\title{
Ultrasonic Adaptive Detection for Aerospace Components with Varying Thickness
}

\author{
Zhen Xiao, Chunguang Xu, Dingguo Xiao, Genyue Peng, and Xinliang Li \\ School of Mechanical Engineering, Beijing Institute of Technology, Beijing 100081, China \\ Correspondence should be addressed to Zhen Xiao; shawzhen86@gmail.com
}

Received 19 October 2016; Accepted 15 January 2017; Published 15 February 2017

Academic Editor: Paolo Boscariol

Copyright ( 2017 Zhen Xiao et al. This is an open access article distributed under the Creative Commons Attribution License, which permits unrestricted use, distribution, and reproduction in any medium, provided the original work is properly cited.

\begin{abstract}
Automatic inspection of microdefects located in the aerospace components is difficult due to the imprecise scanning trajectory, especially for those specimens with varying thickness. In this paper, a new ultrasonic nondestructive testing (NDT) system using the robotic scanning trajectory is constructed for inspecting turbine blades. Additionally, an approach based on the analysis of ultrasonic signals is proposed to calibrate the trajectory; the ultrasonic image based on the threshold function represents the distribution of inner defects when the following gate is used to track the flaw echo. Therefore, the characteristic parameters of the flaw echo signals are easy to be discriminated if the reflection waves are stable in the time domain. Experimental result verified the effective and feasibility of the proposed approach; the distribution of inner defects can be shown with a higher resolution than other NDT methods when robotic orientation is correct at each point of scanning trajectory. Furthermore, the feature signals can be tracked more accurately during the ultrasonic signal processing if the ultrasonic distance was considered as a calibration coefficient of positional matrix. The proposed ultrasonic adaptive detection is adapted to complex geometric structure with a minimum resolution of equivalent diameter of the inner flaw being 0.15 millimeters.
\end{abstract}

\section{Introduction}

The nondestructive testing (NDT) result of aerospace components with varying thickness using traditional approach tended to underperform; sometimes the microdefects cannot to be correctly inspected, due to the imprecise scanning trajectory. In particular, when the ultrasonic incident angles cannot be kept constant as required, the beam axis of probe should be kept normal to the inspected surface; some unnecessary information may be considered by the ultrasonic $\mathrm{C}$-scan imaging and the nondestructive evaluation (NDE) of specimen. Henceforth, the evaluation of integrity and metal fatigue obtained by the signal processing may be misleading. During the automatic inspection, it is necessary to reduce energy attenuation and stabilize the reflection echo so that a more accurate distribution of defects can be distinguished.

Although several methods for automatic inspection at high speeds are proposed and implemented in previous decades, the test results cannot display those microdefects in the specimen because the positional accuracy of probe exceeds the allowed error, especially for those small scale specimens that are sensitive to the errors. Therefore, only noncomplex shapes (plates, cylinders, or cones) can be inspected with a correct NDE result by those semiautomated inspection systems using both mobile and fixed robotic platforms [1]; typically, specific machines such as linear manipulators are designed to inspect identically shaped specimen [2]. Some applications of robotic arms in the NDT field have been published during the last few years and there is a growing interest in using such automatic solutions with many manufacturers within the aerospace sector $[3,4]$.

As an emerging technology to be used in the automatic ultrasonic NDT system, robotic scanning technology receives increasing attention due to its superiority in terms of high repeating accuracy, ease of operation, and accessibility in areas where manual manipulation is not practical $[5,6]$. However, there are some key challenges in the industrial application of robotic NDT system. Firstly, specimens usually are designed identical and will have deviations from the geometric models of Computer-Aided Design (CAD) due to the manufacturing error; this presents a significant challenge for the NDT measurement deployment which should be 
flexible to accommodate these manufacturing issues [7-9]. Secondly, if the probe/specimen are grasped by the robot manipulator, positional accuracy of robotic trajectory would be reduced due to the assembly error. Thus, the orientation of robot should be calibrated and the NDT system should be adaptive to the specimen with modified dimension [10]. Thirdly, due to the error of robotic trajectory, ultrasonic reflection echo may be unstable in the time domain. To eliminate the error and obtain the correct feature signals, the characteristic signals of defects should be captured by a following gate tracking the surface echo wave in real-time.

In this paper, a new calibration method, based on the analysis of ultrasonic signal, is proposed to obtain the correct position and orientation of the robot manipulator. Moreover, the mathematical model of coordinate conversion between the user frame where the probe is fixed and the robotic tool frame is established in Section 2; henceforth, the robotic trajectory can be planned and calibrated with the aid of parameter matrix, where the calibration coefficients are calculated according to the analysis of ultrasonic echo waves. In Section 3, the ultrasonic signal corresponding to the discrete point is collected by the A/D acquisition card, and the ultrasonic distance between the surface echo and the flaw echo in the time domain can be calculated and delivered to the positional matrix, which transform the orientation of robot to meet the requirements of NDT at each discrete point. Only when calibrated, the normal vector of discrete point coincides with the beam axis of probe so that the energy attenuation of reflection echo is limited [11, 12]. Additionally, the following gate is used to track the flaw echo so that a tolerance of incident angle is allowed, to a certain extent, and even the angle has a huge impact on the stability of the ultrasonic echo [13, 14].

As a practical application, a turbine blade with varying thickness is detected by the proposed approach in Section 4 and the distribution of inner defects can be obtained according to the feature signals [15-17]. The feedback from the ultrasonic waves is delivered to the mathematical models so that the robotic orientation can be adjusted to maintain the normal vector of inspected surface that coincides with the beam axis of probe, if the spatial relationship between the specimen and probe is defined. Consequently, the integrity evaluation of blades can be obtained when the data is acquired correctly from experiments. Compared with other calibration approaches, the accuracy of robotic scanning trajectory is enhanced when the proposed ultrasonic adaptive detection is used.

\section{Approaches to Robotic Trajectory Planning for NDT Applications}

Although robotic scanning trajectory is similar to the tool path in the manufacturing process, the constraint for NDT application should be considered when the beam axis of probe determines the orientation of specimen at each discrete point. Considering that some geometric deviations of inspected surface existed in the experiments, it is necessary to

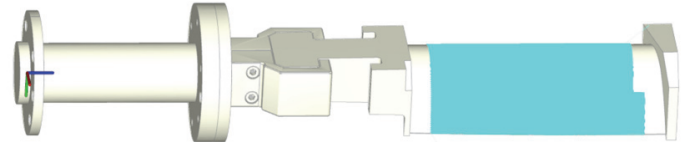

FIGURE 1: The discrete points located on the test surface of specimen's CAD model.

calibrate the robotic trajectory for a high resolution of testing result; moreover, the point cloud data covering the inspected surface should be obtained to satisfy the needs of adaptive detection.

2.1. Trajectory Planning for Specimen. With the aid of the CAD model of specimen and the simulation of tool path based on the computer-aided manufacturing (CAM) algorithm, the green discrete points cover the inspected surface, as shown in Figure 1. Then, points can be extracted from the curved surface of model so that the robotic scanning trajectory can be estimated from those points according to the algorithm of matrix transform. Furthermore, with the aid of parameter matrix which transforms the positional data measured in the coordinate system of specimen to the equivalent data measured in the robotic coordinate systems, robot manipulator can identify the instructions and moves in sequence to perform the automatic inspection.

When the feedback is collected from the ultrasonic waves, the ultrasonic distance between the surface echo and the flaw echo can be delivered to the parameter matrix as a calibration coefficient, as well as the ultrasonic distance between the probe and the surface in the time domain.

2.2. Matrix Transformation. When the geometry information of discrete points is exported from the CAD model, the position and normal vector of single discrete point, located on the test surface, arrange in a row labeled as an array $P_{0}\left[x, y, z, \psi_{x}, \psi_{y}, \psi_{z}\right]$. In order to obtain the orientation and position of robot manipulator, matrix transformation is adopted when the specimen is grasped by the robot. Firstly, the mathematical models of coordinate conversion between the user frame and the robotic tool frame should be calculated based on the matrix transformation. Secondly, the calibration coefficients should be added to the parameter matrixes when the feedback is delivered. Then, the orientation of robot can be adjusted before the automatic inspection, the accuracy of robotic scanning trajectory can be guaranteed, and even some deviations existed in the test specimen when compared with the geometric information of the CAD models. Additionally, the beam axis of probe should be perpendicular to the inspected surface so that energy attenuation of ultrasonic wave is limited and the reflected waveform is steady.

Four coordinate systems consist in the robotic NDT system, the tool frame at the robotic terminate (reference to the coordinate system of specimen) $\{A\}$, auxiliary coordinate system of discrete point $\{B\}$, the world coordinate system of robot $\{W\}$, and the user frame (usually defined as the coordinate system of probe) $\{C\}$ (Figure 2). What is identified by the robot manipulator is the positional data measured in the tool frame or the world coordinate system, an interdeducible 


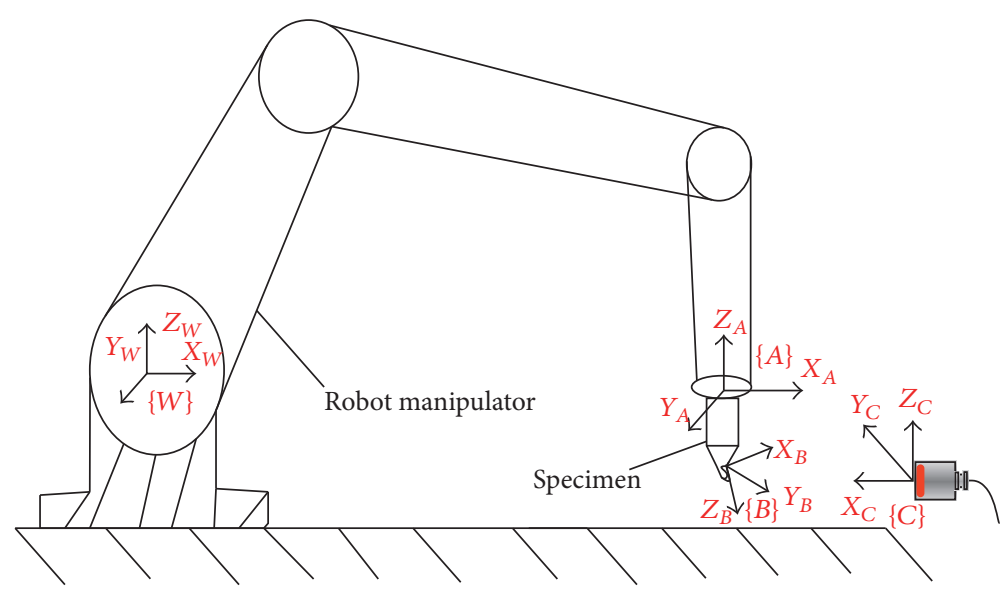

FIGURE 2: Coordinate systems in the robotic NDT system. A: coordinate system of specimen, $B$ : auxiliary coordinate system of discrete point, $C$ : user frame, and $W$ : world coordinate system of robot.

position relationship between them according to the robot kinematics. When the original positional data of discrete points from the CAD model is measured in the coordinate system of specimen, it is necessary to adjust the orientation of robot by the transform of the original position matrix.

The STAUBLI TX90L robot manipulator is used in the experiments; the scanning motion of robot manipulator obeys the $X-Y-Z$ Euler angles rule according to the robotic kinematics. To transform the positional data measured in $\{A\}$ to the equivalent data measured in $\{W\}$, the mathematic model of coordinate conversion is investigated and the calibration coefficient is calculated based on the analysis of ultrasonic echo.

Supposing that the positional vector of discrete point, located on the test surface, has been obtained in $\{W\}$ labeled ${ }^{W} P$, it should rotate and move to the origin of $\{C\}$ located on the beam axis of the probe. The transform matrix ${ }_{C}^{A} T$ can be decomposed into a rotation matrix ${ }_{C}^{A} R$ that describes the orientation of $\{C\}$ with respect to $\{A\}$ and a displacement matrix ${ }^{C} R_{\text {AORG }}$ concluded through the following general formulation:

$$
\begin{aligned}
& { }^{W} P={ }_{C}^{W_{T}}{ }^{C} P={ }_{A}^{W_{B}}{ }_{B}^{A} T_{C}^{B} T{ }^{C} P={ }_{A}^{W_{C}}{ }_{C}^{A} T{ }^{C} P \\
& { }_{A}^{C} T=\left[\begin{array}{cc}
{ }_{A}^{C} R & { }^{C} P_{A O R G} \\
0 & 1
\end{array}\right] \\
& =\left[\begin{array}{cc|cc}
{ }_{B}^{C} R^{T}{ }_{A}^{B} R^{T} & { }_{B}^{C} R^{B} P_{\text {AORG }}+{ }^{C} P_{\text {BORG }} \\
\hline 0 & 0 & 0 & 1
\end{array}\right]
\end{aligned}
$$

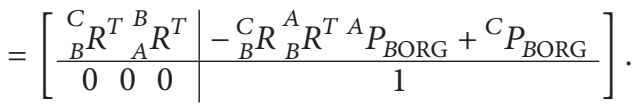

When the origin of $\{B\}$ at the discrete point is labeled ${ }^{A} P_{B O R G}$ relative to $\{A\}$, the rotation matrix ${ }_{B}^{A} R$ can be calculated according to the normal vector components $\left(\psi_{x}, \psi_{y}, \psi_{z}\right)$, and the tangent vector components $\left(o_{x}, o_{y}, o_{z}\right)$ are calculated by the numerical approximation of the adjacent two discrete points; thus, another vector $\left(\omega_{x}, \omega_{y}, \omega_{z}\right)$ can be obtained by the dot product between the normal vector and the tangent vector.

$$
{ }_{B}^{A} R={ }^{A} P^{B} P^{-1}=\left[\begin{array}{lll}
\omega & o & \psi
\end{array}\right]=\left[\begin{array}{lll}
\omega_{X} & o_{X} & \psi_{X} \\
\omega_{Y} & o_{Y} & \psi_{Y} \\
\omega_{Z} & o_{Z} & \psi_{Z}
\end{array}\right] .
$$

Since the beam axis of ultrasonic probe is considered as the orientation constraint of the normal vector of discrete point in $\{B\}$, there will be some overlap between $\{C\}$ and $\{B\}$; therefore, the transform matrix ${ }_{A}^{C} T$ can be simplified to

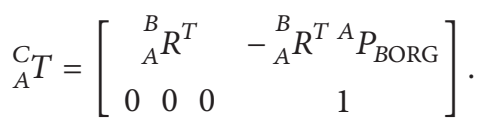

When the specimen is grasped by the robot, the transform matrix ${ }_{A}^{W} T$ describes the orientation of $\{A\}$ relative to $\{W\}$, which can be calculated based on the Denavit-Hartenberg parameters [18-20]. Considering that the robot manipulator has 6 degrees of freedom, the forward kinematics solution is solved and the rotation matrix is given by the following equation:

$$
\begin{aligned}
{ }_{A}^{W} T & ={ }_{6}^{0} T={ }_{1}^{0} T_{2}^{1} T_{3}^{2} T_{4}^{3} T_{5}^{4} T_{6}^{5} T \\
& =\left[\begin{array}{cccc}
R_{11} & R_{12} & R_{13} & R_{14} \\
R_{21} & R_{22} & R_{23} & R_{24} \\
R_{31} & R_{32} & R_{33} & R_{34} \\
0 & 0 & 0 & 1
\end{array}\right] .
\end{aligned}
$$

Where the components of rotation matrix can be solved as follows:

$$
\begin{aligned}
R_{11}= & c_{1}\left[c_{23}\left(c_{4} c_{5} c_{6}-s_{4} s_{6}\right)-s_{23} s_{5} c_{6}\right] \\
& -s_{1}\left[s_{4} c_{5} c_{6}+c_{4} s_{6}\right] \\
R_{21}= & s_{1}\left[c_{23}\left(c_{4} c_{5} c_{6}-s_{4} s_{6}\right)-s_{23} s_{5} c_{6}\right] \\
& +c_{1}\left(s_{4} c_{5} c_{6}+c_{4} s_{6}\right)
\end{aligned}
$$




$$
\begin{aligned}
R_{31}= & s_{23}\left(s_{4} s_{6}-c_{4} c_{5} c_{6}\right)-c_{23} s_{5} c_{6} \\
R_{12}= & c_{1}\left[-c_{23}\left(c_{4} c_{5} s_{6}+s_{4} c_{6}\right)+s_{23} s_{5} s_{6}\right] \\
& +s_{1}\left(s_{4} c_{5} s_{6}-c_{4} c_{6}\right) \\
R_{22}= & s_{1}\left[-c_{23}\left(c_{4} c_{5} s_{6}+s_{4} c_{6}\right)+s_{23} s_{5} s_{6}\right] \\
& -c_{1}\left(s_{4} c_{5} s_{6}-c_{4} c_{6}\right) \\
R_{32}= & s_{23}\left(s_{4} c_{6}+c_{4} c_{5} s_{6}\right)+c_{23} s_{5} s_{6} \\
R_{13}= & c_{1}\left(s_{23} c_{5}+c_{23} c_{4} s_{5}\right)-s_{1} s_{4} s_{5} \\
R_{23}= & s_{1}\left(s_{23} c_{5}+c_{23} c_{4} s_{5}\right)+c_{1} s_{4} s_{5} \\
R_{33}= & c_{23} c_{5}-s_{23} c_{4} s_{5} \\
R_{14}= & c_{1}\left[a_{1}+a_{2} c_{2}+d_{4} s_{23}+d_{6}\left(s_{23} c_{5}+c_{23} c_{4} s_{5}\right)\right] \\
& -s_{1}\left[d_{3}+d_{6} s_{4} s_{5}\right] \\
R_{24}= & s_{1}\left[a_{1}+a_{2} c_{2}+d_{4} s_{23}+d_{6}\left(s_{23} c_{5}+c_{23} c_{4} s_{5}\right)\right] \\
& +c_{1}\left[d_{3}+d_{6} s_{4} s_{5}\right] \\
R_{34}= & d_{4} c_{23}+d_{6}\left(c_{23} c_{5}-s_{23} c_{4} s_{5}\right)-a_{2} s_{2} .
\end{aligned}
$$

In order to be observed more clearly, some parameters are simplified as

$$
\begin{aligned}
c_{i} & =\cos \theta_{i} ; \\
s_{i} & =\sin \theta_{i} ; \\
c_{i j} & =\cos \left(\theta_{i}+\theta_{j}\right) ; \\
s_{i j} & =\sin \left(\theta_{i}+\theta_{j}\right) .
\end{aligned}
$$

The equation of Euler angle is defined according to the robotic kinematics:

$$
\begin{aligned}
& R_{X^{\prime} Y^{\prime} Z^{\prime}}(\alpha, \beta, \gamma)=R_{X}(\alpha) R_{Y}(\beta) R_{Z}(\gamma) \\
& \quad=\left[\begin{array}{ccc}
c \beta c \gamma & -c \beta s \gamma & s \beta \\
s \alpha s \beta c \gamma+c \alpha s \gamma & -s \alpha s \beta s \gamma+c \alpha c \gamma & -s \alpha c \beta \\
-c \alpha s \beta c \gamma+s \alpha s \gamma & c \alpha s \beta s \gamma+s \alpha c \gamma & c \alpha c \beta
\end{array}\right] .
\end{aligned}
$$

In the matrix (7), $\alpha, \beta$, and $\gamma$ are the Euler angles which indicate the change of orientation between the latter point and the former; the parameter such as $\cos \alpha$ and $\sin \alpha$ is abbreviated to $c \alpha$ and $s \alpha$.

Substituting (2) into (7), the Euler angles of each point located on the robotic scanning trajectory can be calculated as follows:

$$
\begin{aligned}
& \beta=a \tan 2\left(\psi_{x}, \sqrt{\omega_{x}^{2}+o_{x}^{2}}\right) \\
& \alpha=a \tan 2\left(\frac{-\psi_{y}}{\cos \beta}, \frac{\psi_{z}}{\cos \beta}\right) \\
& \gamma=a \tan 2\left(\frac{-o_{x}}{\cos \beta}, \frac{\omega_{x}}{\cos \beta}\right) .
\end{aligned}
$$

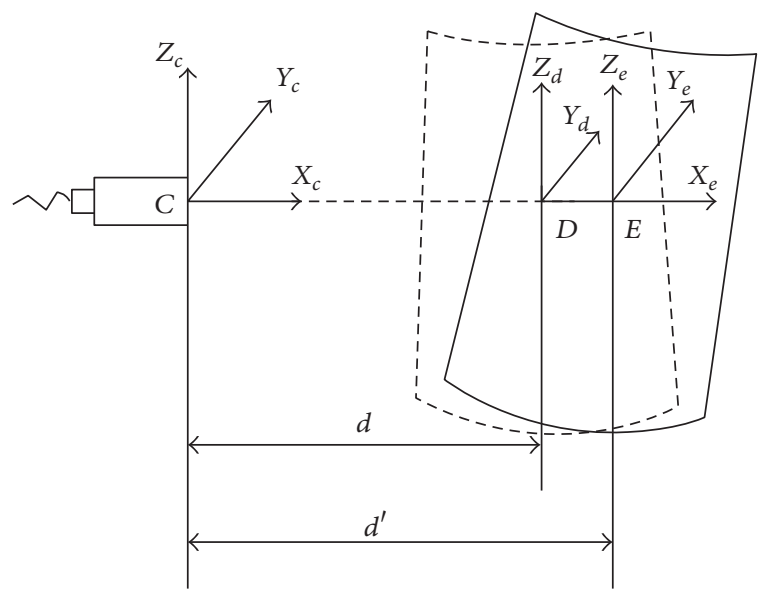

FIGURE 3: Ultrasonic distance in the experiment.

Henceforth, the transform matrix ${ }_{C}^{W} T$ can be concluded

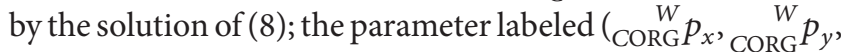
$\left.\underset{\mathrm{CORG}}{W} p_{z}, \alpha, \beta, \gamma\right)$ is obtained as required of the robot controller.

2.3. Calibration by the Ultrasonic Signals. A constant ultrasonic entry angle is necessary during the automatic NDT of test object, and each discrete point of robotic trajectory requires a correct position and orientation. In this case, the energy attenuation of reflection echo is limited and stable ultrasonic waves are guaranteed. On the other hand, there are some deviations of robotic position and orientation existing in the test object due to the assembly errors, frictions, abrasions, and so on [21-23]. The spatial relationship between the robotic tool frame and the user frame should be estimated with the aid of coordinate conversion algorithm. Furthermore, several typical points can be tracked according to the ultrasonic signals in the time domain, and the signals can be analyzed to estimate the correct orientation and position of the specimen.

As shown in Figure 3, the specified distance between the inspected surface and the probe is labeled $d$; if the robotic scanning trajectory deviated from the specified position, the actual distance labeled $d^{\prime}$ can be calculated according to the ultrasonic signals in the time domain. Moreover, the deflection angle can be estimated based on the signal analysis and delivered to the parameter matrix as a calibration coefficient. The system errors can be reduced if the deflection angle was compensated and the robotic orientation has been adjusted.

Supposing the deviation value between the specified distance $d$ and the actual distance $d^{\prime}$ is labeled $\Delta d=d^{\prime}-d$, the actual position of discrete point on the test surface and the components of deviation can be solved by the following general formulation:

$$
\begin{aligned}
\Delta d & =\sqrt{\Delta d_{x}^{2}+\Delta d_{y}^{2}+\Delta d_{z}^{2}} \\
\Delta d_{x} & =\omega_{x} \cdot \Delta d+o_{x} \cdot \Delta d+\psi_{x} \cdot \Delta d
\end{aligned}
$$




$$
\begin{aligned}
& \Delta d_{y}=\omega_{y} \cdot \Delta d+o_{y} \cdot \Delta d+\psi_{y} \cdot \Delta d \\
& \Delta d_{z}=\omega_{z} \cdot \Delta d+o_{z} \cdot \Delta d+\psi_{z} \cdot \Delta d
\end{aligned}
$$

$$
\begin{aligned}
& { }^{D} P={ }_{E} T^{E} P=\left[\begin{array}{cccc}
C \beta^{\prime} C \gamma^{\prime} & -C \beta^{\prime} s \gamma^{\prime} & s \beta^{\prime} & \Delta d_{x} \\
s \alpha^{\prime} s \beta^{\prime} c \gamma^{\prime}+c \alpha^{\prime} s \gamma^{\prime} & -s \alpha^{\prime} s \beta^{\prime} s \gamma^{\prime}+c \alpha^{\prime} c \gamma^{\prime} & -s \alpha^{\prime} c \beta^{\prime} & \Delta d_{y} \\
-c \alpha^{\prime} s \beta^{\prime} c \gamma^{\prime}+s \alpha^{\prime} s \gamma^{\prime} & c \alpha^{\prime} s \beta^{\prime} s \gamma^{\prime}+s \alpha^{\prime} c \gamma^{\prime} & c \alpha^{\prime} c \beta^{\prime} & \Delta d_{z} \\
0 & 0 & 0 & 1
\end{array}\right]\left[\begin{array}{c}
x+\Delta d_{x} \\
y+\Delta d_{y} \\
z+\Delta d_{z} \\
1
\end{array}\right]=\left[\begin{array}{c}
x \\
y \\
z \\
1
\end{array}\right] \\
& F=\left\{\begin{array}{l}
f_{1}\left(\alpha^{\prime}, \beta^{\prime}, \gamma^{\prime}\right) \\
f_{2}\left(\alpha^{\prime}, \beta^{\prime}, \gamma^{\prime}\right) \\
f_{3}\left(\alpha^{\prime}, \beta^{\prime}, \gamma^{\prime}\right)
\end{array}\right. \\
& =\left\{\begin{array}{l}
c \beta^{\prime} c \gamma^{\prime}\left(x+\Delta d_{x}\right)-c \beta^{\prime} s \gamma^{\prime}\left(y+\Delta d_{y}\right)+s \beta^{\prime}\left(z+\Delta d_{z}\right)+\Delta d_{x}-x=0 \\
\left(s \alpha^{\prime} s \beta^{\prime} c \gamma^{\prime}+c \alpha^{\prime} s \gamma^{\prime}\right)\left(x+\Delta d_{x}\right)+\left(-s \alpha^{\prime} s \beta^{\prime} s \gamma^{\prime}+c \alpha^{\prime} c \gamma^{\prime}\right)\left(y+\Delta d_{y}\right)-s \alpha^{\prime} c \beta^{\prime}\left(z+\Delta d_{z}\right)+\Delta d_{y}-y=0 \\
\left(-c \alpha^{\prime} s \beta^{\prime} c \gamma^{\prime}+s \alpha^{\prime} s \gamma^{\prime}\right)\left(x+\Delta d_{x}\right)+\left(c \alpha^{\prime} s \beta^{\prime} s \gamma^{\prime}+s \alpha^{\prime} c \gamma^{\prime}\right)\left(y+\Delta d_{y}\right)-c \alpha^{\prime} c \beta^{\prime}\left(z+\Delta d_{z}\right)+\Delta d_{z}-z=0 .
\end{array}\right.
\end{aligned}
$$

When the specified robotic orientation and position have been obtained from the planned trajectory, the deviation, usually considered as an system error, should be taken into consideration by the parameter matrix; the changes of Euler angles are labeled as $\alpha^{\prime}, \beta^{\prime}$, and $\gamma^{\prime}$, given by the transform matrix.
The compensation of Euler angles can be calculated if the specified position $(x, y, z)$ and deviation value $\left(\Delta d_{x}, \Delta d_{y}, \Delta d_{z}\right)$ are obtained by the analysis of ultrasonic signals in the time domain, considering the transform matrix ${ }_{E}^{D} T$ is an orthogonal matrix and the value of Euler angles should be changed on a small scale; the adjusted Euler angles $\alpha^{\prime}, \beta^{\prime}$, and $\gamma^{\prime}$ can be solved by the numerical iteration, assuming the deviation value is approaching zero.

Suppose that a function $\Phi\left(\alpha^{\prime}, \beta^{\prime}, \gamma^{\prime}\right)=\sum_{i=1}^{3}\left[f_{i}\left(\alpha^{\prime}, \beta^{\prime}\right.\right.$, $\left.\left.\gamma^{\prime}\right)\right]^{2}$ is used to obtain the optimal solution of robotic orientation. During the iteration, the gradient of function is $G_{k}=\left(\partial \Phi / \partial \alpha^{\prime}, \partial \Phi / \partial \beta^{\prime}, \partial \Phi / \partial \gamma^{\prime}\right)_{\alpha^{\prime}=\alpha^{\prime(k)}, \beta^{\prime}=\beta^{\prime(k)}, \gamma^{\prime}=\gamma^{\prime(k)}}$; if $G_{k}$ is assigned a valid value, there would exist a point $\Phi\left(\alpha^{\prime(1)}, \beta^{\prime(1)}, \gamma^{\prime(1)}\right)$ smaller than the initial value $\Phi\left(\alpha^{\prime(0)}\right.$, $\left.\beta^{\prime(0)}, \gamma^{\prime(0)}\right)$. When it is approaching the specified point along the gradient vector, the limiting condition $\left\|G_{k}\right\|<\varepsilon$ decides the iteration cycle until a constraint $\varepsilon$ is given to stop the iteration; otherwise, the solution should be iterated until the $\min _{\lambda>0} \Phi\left(\alpha^{\prime(k)}-\lambda G_{k}, \beta^{\prime(k)}-\lambda G_{k}, \gamma^{\prime(k)}-\lambda G_{k}\right)$ has been solved. Therefore, the function $\lambda\left(\alpha^{\prime}, \beta^{\prime}, \gamma^{\prime}\right)$ is the optimal solution approach to the correct robotic orientation.

The robotic orientation can be adjusted when the parameter matrix $\left[x+\Delta d x, y+\Delta d y, z+\Delta d z, \alpha+\alpha^{\prime}, \beta+\beta^{\prime}, \gamma+\right.$ $\gamma^{\prime}$ ] has been solved, and the coordinate of specimen can be identified by the robot manipulator if the typical points of robotic scanning trajectory have been calibrated. Meanwhile, the system error can be eliminated if the deviation values $\Delta d x, \Delta d y$, and $\Delta d z$ have been compensated by the robotic scanning trajectory.

2.4. Simulation and Analysis. Figure 4 gives the point cloud data of planned trajectory for a turbine blade, the data is delivered to the robot controller, the spatial motion trail of the robot has been simulated in the MasterCAM, as shown in Figure 5, and the discrete point data can be exported for constructing parameter matrix and transform the robotic orientation as planned to satisfy the NDT requirements.

The robotic trajectory has been monitored and the positional data was exported real-time during the test, as well as the ultrasonic signals that offer the necessary information for calibration. Moreover, the detection image is shown to the inspector and the distribution of flaws can be displayed synchronously.

\section{Ultrasonic Adaptive Detection with Signal Processing}

The specimen is grasped by the robot manipulator and moves in sequence to pass the specified point, and the ultrasonic wave is excited at the same time so that the reflection echo of inspected object can be collected by the A/D acquisition card. Hence, the positional data and ultrasonic signals can be delivered to the industrial computer synchronously. Each point of robotic trajectory would be assigned an ultrasonic signal, which represents the integrity of specimen at the corresponding position; the detection process and components of ultrasonic NDT system can be described in Figure 6.

The ultrasonic distance $L_{S}$, between the surface echo and bottom echo in the time domain, usually changes in the experiment due to the varying thickness of specimen, and even the robotic scanning trajectory has been calibrated. A following gate is used to track the flaw echo wave, which keeps a constant distance $L_{f}$ from the surface echo waves if the detection region is designated by the inspector. Therefore, a positional tolerance of flaw echo can be allowed to a certain 


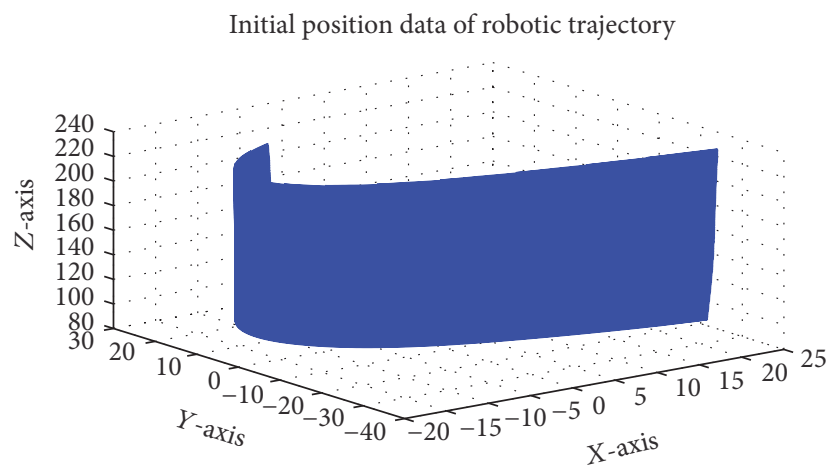

FIgURE 4: The discrete point cloud of robotic trajectory.

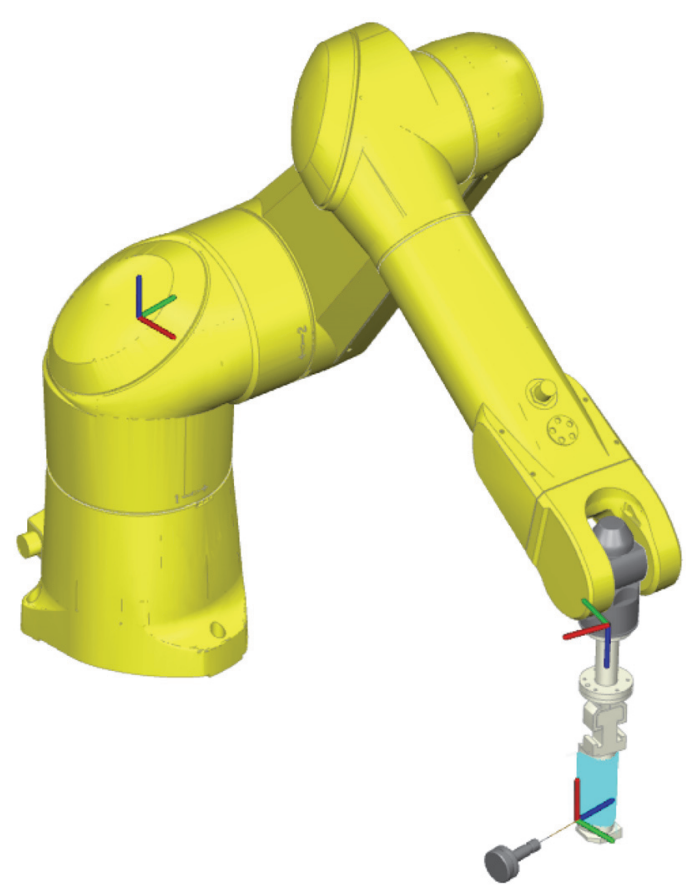

Figure 5: The simulation of robotic trajectory.

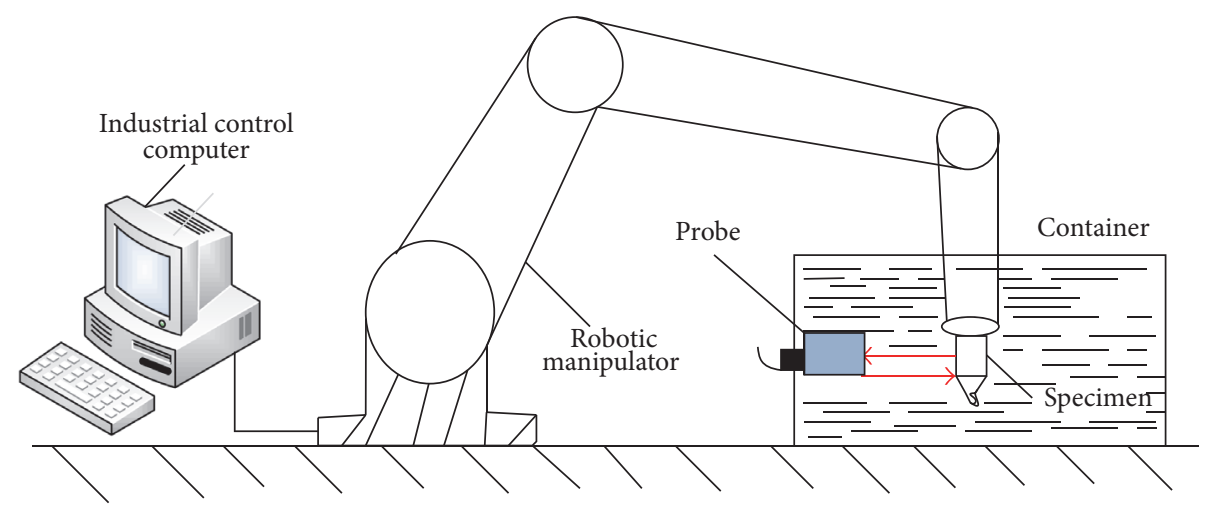

FIGURE 6: The ultrasonic NDT system. 


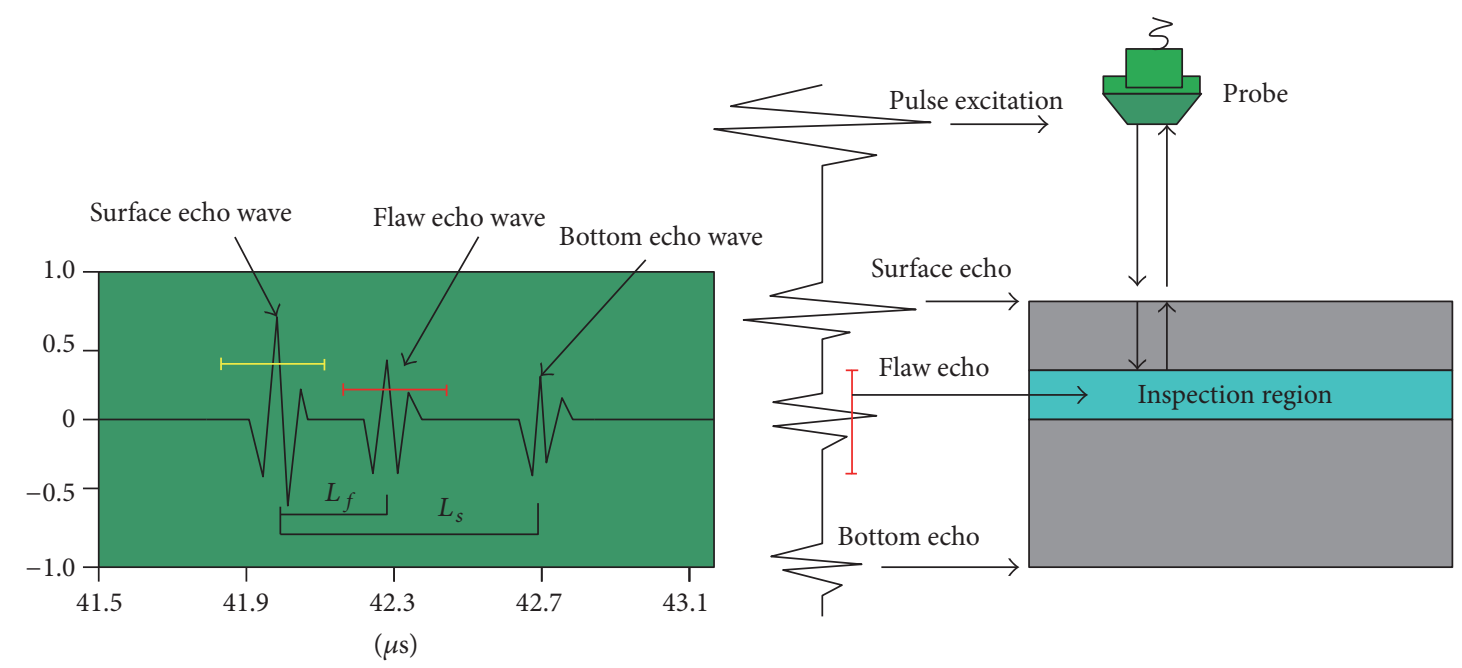

Figure 7: The following gate used to track echo waves.

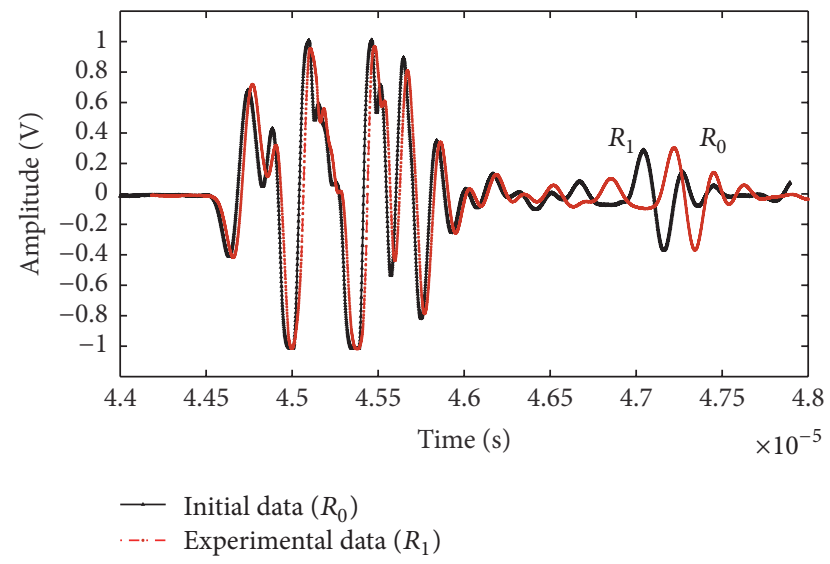

Figure 8: The feature signal of flaw echo in the time domain.

extent during the autonomic NDT at high speeds, especially for those specimens with varying thickness that is challenge to collect the correct signals of flaw echo using the traditional methods. As shown in Figure 7, the distance $L_{f}$ between the surface echo and the flaw echo can be calculated based on the ultrasonic signals in the time domain, and the feature signal of flaw echo can be captured if the ultrasonic wave is tracked by a following gate, as well as the thickness of specimen obtained from the distance $L_{S}$ between the surface echo and bottom echo.

When the robotic scanning trajectory has been calibrated, the position of surface echo in the time domain, which keeps a constant distance from the excitation source, is stable and offers a reference to the position of flaw echo in the time domain. Similarly, the wave crest of flaw echo can be tracked by a following gate, which is assigned before the experiments according to the inspection region.

As shown in Figure 8, when the discrete point on the inspected surface kept a consistent distance from the probe, the reflection echo would be stable in the time domain, manifesting as the propagation time of reflection echo focus at $44.5 \mu$ s or so. During the ultrasonic testing, the position of flaw echo may changes because the distribution of defects is diverse at different discrete point. Similarly, the amplitude of flaw echo changes in the experiment so that the character of integrity at different area can be estimated by the ultrasonic signals, and a grey value that transformed from the amplitude is assigned to the corresponding point. The position of flaw echo $R_{1}$ in the time domain is earlier than the flaw echo $R_{0}$, representing that the location of defect is near the surface and the thickness of specimen is changed when the distance between the surface echo and bottom echo is shorter than the initial length.

The robotic scanning trajectory is continuous and covers the inspected surface so that the position accuracy is enough during the automatic inspection; the trajectory can be calibrated by the ultrasonic signals if the normal vector of typical point is aligned with the beam axis of probe; then, the relationship between the coordinate system of specimen and the robotic tool frame can be defined. Each discrete point of the robotic scanning trajectory corresponding to an ultrasonic signal, which is excited when the robot manipulator arrived at the specified point, and the multithreaded programming guarantee that the positional data and corresponding ultrasonic signal are matched correctly so that the inner defects can be distinguished from the ultrasonic C-scan imaging.

\section{Experimental Results}

A turbine blade is detected using the proposed approach adaptive to the specimens with varying thickness, and the feature signals of flaw echo can be discriminated more accurately; the resolution of inspection imaging would be enhanced due to the correct relationship between the discrete point and the ultrasonic signal, guaranteed by the ultrasonic NDT system. At the same time, the robotic scanning trajectory should be calibrated to keep the normal vector of test surface coincided with the beam axis of probe so that 


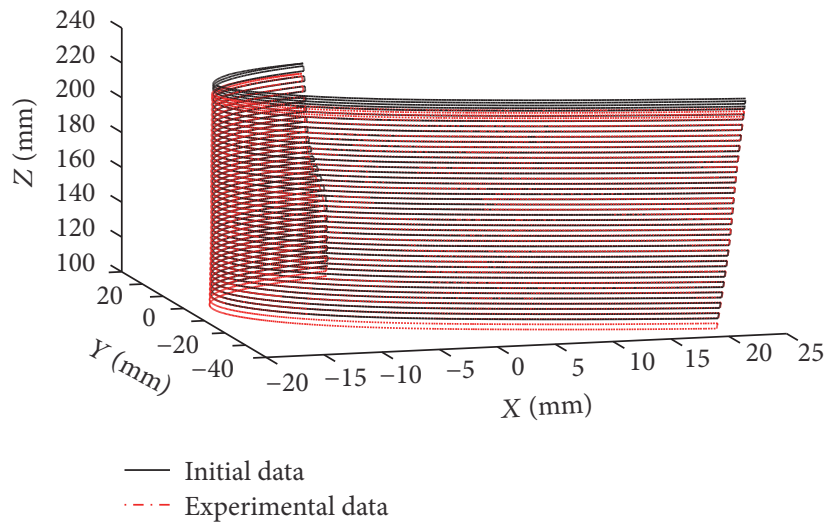

(a) The initial and transformed robotic scanning trajectory in the experiment

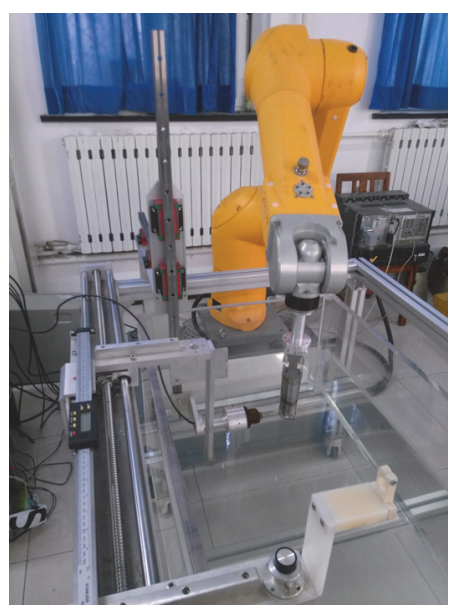

(b) The robotic scanning motion in the experiment

FIGURE 9: The robotic scanning trajectory in the experiment.

the ultrasonic signal is stable and easy to be tracked by the following gate.

Figure 9(a) gives the initial positional data, measured in the coordinate system of specimen, from the CAD model of test specimen. Considering that the experimental data should be transformed to be measured in the same system so that scanning trajectory can be observed and provided to the inspector for an evaluation, the trajectory should pass the identical point, which keeps a constant distant from the origin of probe, to stabilize the reflection echo. The robotic scanning motion is shown as in Figure 9(b).

Table 1 listed the ultrasonic distance between the probe and the test surface in the time domain; the ultrasonic wave is stable during the NDT application, which is necessary for the detection precision of experiment result. The peak value of reflection waves is labeled $H$, focused on the region which in the time domain is $44 \mu \mathrm{s}$ or so. The minimum resolution of equivalent diameter of inner flaw can be calculated from the distance between the adjacent points on the robotic trajectory, which is $0.15 \mathrm{~mm}$ as required.

There are several reflection echoes obtained from the experiments, shown in Figure 10. In comparison with the reflection echo wave 1, the amplitudes of reflection echo wave 2 and the flaw echo are changed due to the different position of discrete points on the test surface, as shown in Figure 10(a); similarly, the positions of bottom echoes are changed due to the varying thickness of specimen, as described by the surface echoes 1 and 2 and the reflection echeos 1 and 2. When the position and amplitude of ultrasonic signals are changed, the following gate should be used to track the region of interest; then, the character of defects can be evaluated if each discrete point has assigned an ultrasonic wave received by the A/D acquisition card.

The experiment demonstrates that the propagation time of surface echo is stable at different points in the time domain, about $44.5 \mu \mathrm{s}$ as shown in Figure 10(b). If the ultrasonic distance between the probe and inspected surface is consistent, the robotic scanning trajectory has been calibrated.
TABLE 1: Ultrasonic echo waves at different points of robotic scanning trajectory.

\begin{tabular}{lcccc}
\hline $\begin{array}{l}\text { Experiment } \\
\text { number }\end{array}$ & $d / \mu \mathrm{s}$ & $L_{f} / \mu \mathrm{s}$ & $L_{S} / \mu \mathrm{s}$ & $H / \mathrm{mV}$ \\
\hline 1 & 43.3 & 1.9 & 3.1 & 1.5 \\
2 & 43.3 & 2.0 & 3.5 & 1.22 \\
3 & 43.5 & 1.6 & 3.3 & 1.3 \\
4 & 42.4 & 1.8 & 4.0 & 1.4 \\
5 & 42.7 & 2.0 & 4.1 & 1.54 \\
$\vdots$ & $\vdots$ & $\vdots$ & $\vdots$ & \\
33 & 42.7 & 2.0 & 4.0 & 1.4 \\
34 & 43.9 & 2.4 & 4.0 & 1.3 \\
35 & 44.2 & 2.3 & 3.3 & 1.0 \\
36 & 44.2 & 1.3 & 3.4 & 1.2 \\
\hline
\end{tabular}

When the wave crest of flaw echo exceeds the threshold that represents the character of integrity in the detection region, the position in the time domain and peak value of flaw echo should be recorded synchronously and delivered to the computer; then, the distribution of flaw can be shown as the ultrasonic C-scan imaging, where each pixel has a grey value assigned according to the peak value of flaw echo.

Four embedded holes in the turbine blade are inspected; the diameters of holes are $2 \mathrm{~mm}, 1.5 \mathrm{~mm}, 1 \mathrm{~mm}$, and $0.5 \mathrm{~mm}$, respectively. If the position and orientation of robotic trajectory are inaccurate or inconsistent during the automatic detection, usually calibrated with the traditional measuring instrument such as a gauge, the character of inner defects may be mismatched; then, some image distortion would occur in the inspection image, as shown in Figure 11(a), and some unnecessary information is recorded by the following gate so that the $0.5 \mathrm{~mm}$ hole cannot be identified from the ultrasonic imaging. Conversely, the distribution of inner flaws can be observed clearly if the trajectory has been adjusted by the 

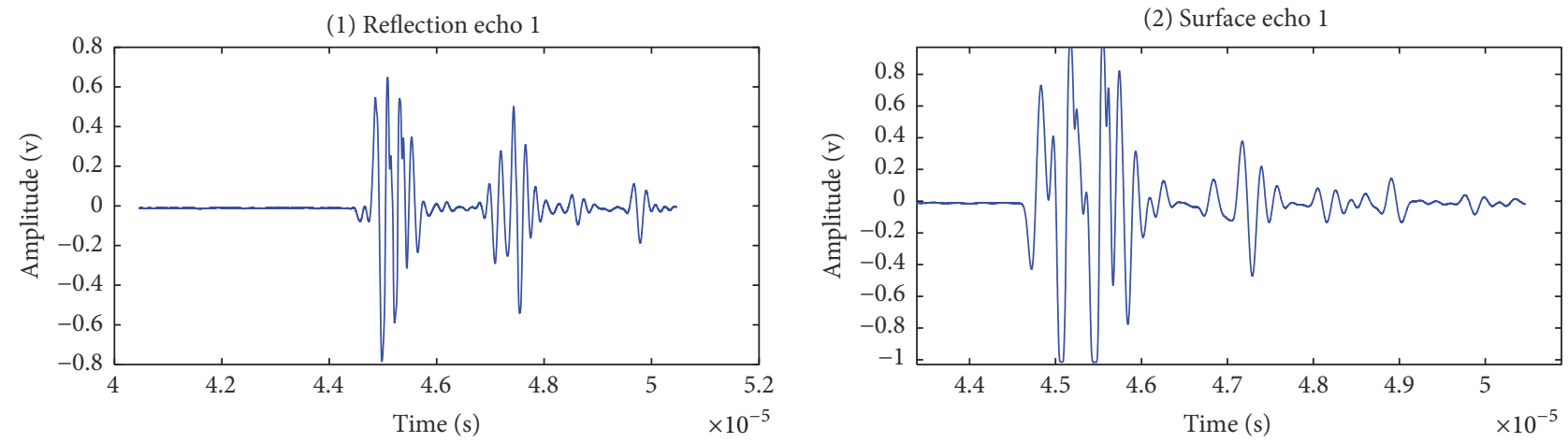

(3) Reflection echo 2

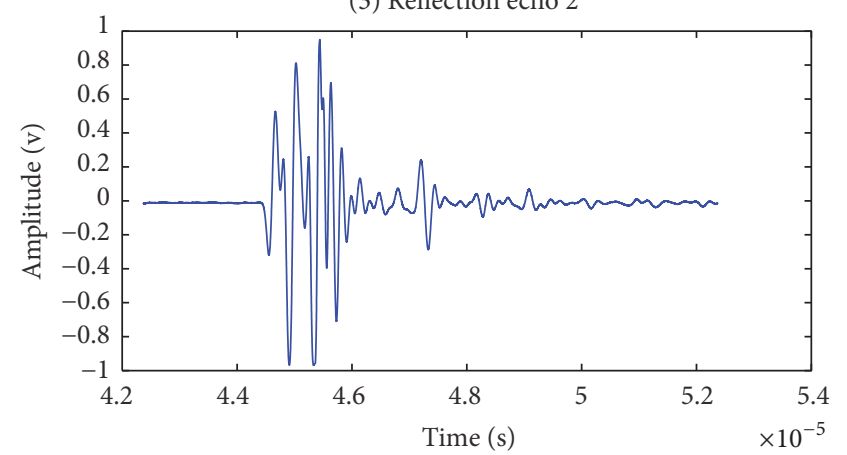

(4) Surface echo 2

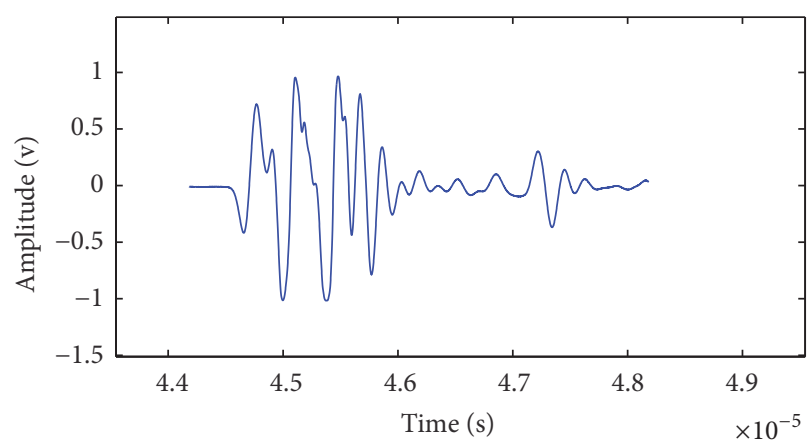

(a) The ultrasonic waves from NDT experiments

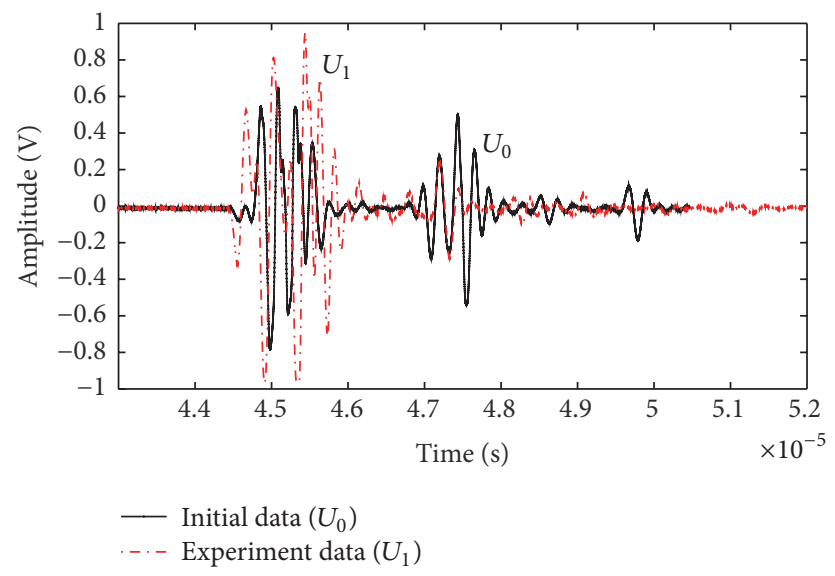

(b) The comparison of ultrasonic waves at different points

FIGURE 10: The ultrasonic signals in the experiments.

proposed approach, as shown in Figure 11(b). The artificial defects can be discriminated from other inspection regions according to the different grey value assigned according to the ultrasonic signals, shown as a colorful region to be distinguished easily.

\section{Conclusions}

In this study, a comprehensive study of ultrasonic adaptive detection for aerospace components is conducted, the complex geometric specimen with varying thickness can be automatically inspected more precisely if the trajectory is calibrated based on the analysis of ultrasonic echo. There is a far-reaching impact on the manufacturing industry and improving the security of key parts and components in the aeronautical system.

(1) A calibration approach of robotic scanning trajectory is proposed to enhance the detection precision when the position and orientation of specimen were correct at each discrete point as required.

(2) The mathematic model of coordinate conversion between the coordinate of specimen and the robotic tool frame is proposed to take the feedback from the ultrasonic distance into consideration; the experiment results demonstrating the calibrated trajectory have more accuracy than the planned trajectory. 


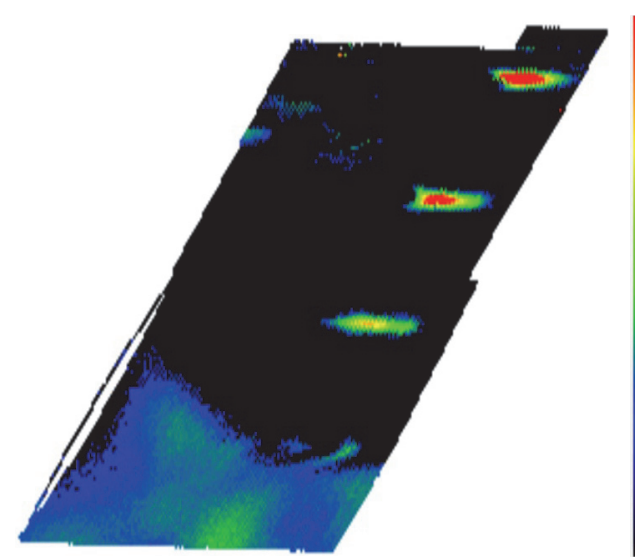

(a) Ultrasonic C-scan imaging using traditional calibration

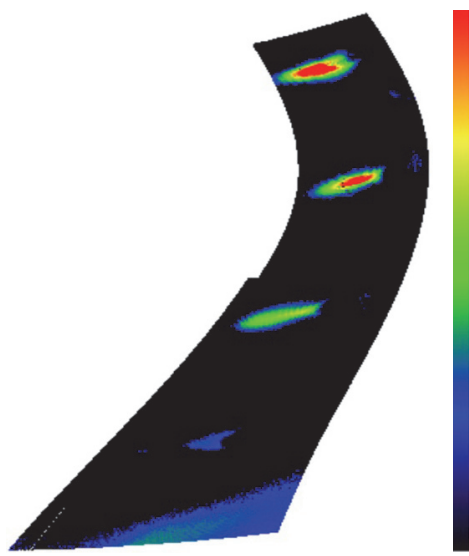

(b) Ultrasonic C-scan imaging using proposed approach

FIGURE 11: The ultrasonic C-scan imaging of turbine blades.

(3) The ultrasonic waveform is stable and reliable in the time domain if the trajectory has been calibrated and meets the requirements of consistent incident angles and ultrasonic distance between the inspected surface and the probe.

(4) The flaw echo can be tracked by the following gate more convenient when the ultrasonic signals were acquired synchronously with the robotic positional data; then, the change value between the surface echo and flaw echo can be calculated according to the feature signals in the time domain.

(5) The imaging distortion is reduced in the ultrasonic C-scan imaging with the aid of correctly matching between the robotic scanning trajectory and the feature signal of flaw echo; the resolution of inspection result is enhanced due to the proposed calibration approach.

The ultrasonic adaptive detection with proposed calibration approach can improve the detection precision and provide an important reference to the inspector for an evaluation about the integrity of the specimen.

\section{Competing Interests}

The authors declare that there is no conflict of interests regarding the publication of this paper.

\section{Acknowledgments}

This research work is supported by the project of Advanced NC Machine Tools \& Basic Manufacturing Equipment, Ministry of Science \& Technology of China (Grant no. 2014ZX04012015), and the National Natural Science Foundation of China (Grant no. 51335001).

\section{References}

[1] M. Tavakoli, L. Marques, and A. T. D. Almeida, "Development of an industrial pipeline inspection robot," Industrial Robot, vol. 37, no. 3, pp. 309-322, 2010.

[2] J. Shang, B. Bridge, T. Sattar, S. Mondal, and A. Brenner, "Development of a climbing robot for inspection of long weld lines," Industrial Robot, vol. 35, no. 3, pp. 217-223, 2008.

[3] M. R. Pedersen, L. Nalpantidis, R. S. Andersen et al., "Robot skills for manufacturing: from concept to industrial deployment," Robotics and Computer-Integrated Manufacturing, vol. 37, pp. 282-291, 2016.

[4] W. H. Weber, H. D. Mair, D. Jansen, and L. Lombardi, "Advances in inspection automation," Review of Progress in Quantitative Non-Destructive Evaluation, vol. 32, no. 1511, pp. 1654-1661, 2013.

[5] K. P. Liu, B. L. Luk, F. Tong, and Y. T. Chan, "Application of service robots for building NDT inspection tasks," Industrial Robot, vol. 38, no. 1, pp. 58-65, 2011.

[6] J. Shang, T. Sattar, S. Chen, and B. Bridge, "Design of a climbing robot for inspecting aircraft wings and fuselage," Industrial Robot, vol. 34, no. 6, pp. 495-502, 2007.

[7] T. Messay, R. Ordóñez, and E. Marcil, “Computationally efficient and robust kinematic calibration methodologies and their application to industrial robots," Robotics and ComputerIntegrated Manufacturing, vol. 37, article no. 1350, pp. 33-48, 2016.

[8] T. P. Sattar and A.-A. Brenner, "Robotic system for inspection of test objects with unknown geometry using NDT methods," Industrial Robot, vol. 36, no. 4, pp. 340-343, 2009.

[9] R. Summan, S. Pierce, G. Dobie, J. Hensman, and C. Macleod, "Practical constraints on real time Bayesian filtering for NDE applications," Mechanical Systems and Signal Processing, vol. 42, no. 1-2, pp. 181-193, 2014.

[10] M. Ross, D. Klose, and H. Jarod, "Conceptual design of NDT FIFLD inspection system," IMECE 2008: Safety Engineering, Risk Analysis And Reliability Methods, vol. 1, no. 6, pp. 335-338, 2009.

[11] L. Xue, Y. Zou, J. Huang, J. Huang, X. Tao, and Y. Hu, “Constant speed control for complex cross-section welding using robot 
based on angle self-test," Chinese Journal of Mechanical Engineering, vol. 27, no. 2, pp. 260-268, 2014.

[12] Y. Miao, Y. Liu, H. Ma, and H. Jin, "The pose estimation of mobile robot based on improved point cloud registration," International Journal of Advanced Robotic Systems, vol. 13, no. 52, pp. 342-350, 2016.

[13] Z. Lu, C. Yang, D. Qin, and Y. Luo, "Estimating the parameters of ultrasonic echo signal in the Gabor transform domain and its resolution analysis," Signal Processing, vol. 120, pp. 607-619, 2016.

[14] N. Kashiri, M. Laffranchi, D. G. Caldwell, and N. G. Tsagarakis, "Dynamics and control of an anthropomorphic compliant arm equipped with friction clutches," IEEE/ASME Transactions on Mechatronics, vol. 21, no. 2, pp. 694-707, 2016.

[15] J. A. Sánchez-Durán, J. A. Hidalgo-López, J. CastellanosRamos, Ó. Oballe-Peinado, and F. Vidal-Verdú, "Influence of errors in tactile sensors on some high level parameters used for manipulation with robotic hands," Sensors, vol. 15, no. 8, pp. 20409-20435, 2015.

[16] D. Stavrou, D. G. Eliades, C. G. Panayiotou, and M. M. Polycarpou, "Fault detection for service mobile robots using model-based method," Autonomous Robots, vol. 40, no. 2, pp. 383-394, 2016.

[17] A. S. Rezazadeh, H. R. Koofigar, and S. Hosseinnia, "Adaptive fault detection and isolation for a class of robot manipulators with time-varying perturbation," Journal of Mechanical Science and Technology, vol. 29, no. 11, pp. 4901-4911, 2015.

[18] H.-S. Wang, D.-Y. Zhan, P.-L. Huang, and W.-F. Chen, "Inverse kinematics of a heavy duty manipulator with 6-DOF based on dual quaternion," Journal of Central South University, vol. 22, no. 7, pp. 2568-2577, 2015.

[19] B. Zhao, C. Li, T. Ma, and Y. Li, "Multiple faults detection and isolation via decentralized sliding mode observer for reconfigurable manipulator," Journal of Electrical Engineering \& Technology, vol. 10, no. 6, pp. 2393-2405, 2015.

[20] K. S. Fu, R. C. Gonzalez, and C. X. G. Lee, Robotics: Control, Sensing, Vision, and Intelligence, McGraw-Hill, 1987.

[21] C. Kim, D. Espalin, A. Cuaron et al., "Cooperative tool path planning for wire embedding on additively manufactured curved surfaces using robot kinematics," Journal of Mechanisms and Robotics, vol. 7, no. 2, Article ID 021003, 2015.

[22] Z. Lu, C. Xu, D. Xiao, and F. Meng, "Nondestructive testing method for curved surfaces based on the multi-gaussian beam model," Journal of Nondestructive Evaluation, vol. 34, no. 4, pp. 30-39, 2015.

[23] Z. Lu, C. Xu, Q. Pan, F. Meng, and X. Li, "Automatic method for synchronizing workpiece frames in twin-robot nondestructive testing system," Chinese Journal of Mechanical Engineering, vol. 28, no. 4, pp. 860-868, 2015. 


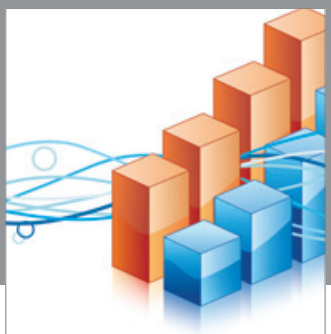

Advances in

Operations Research

vatem alat4

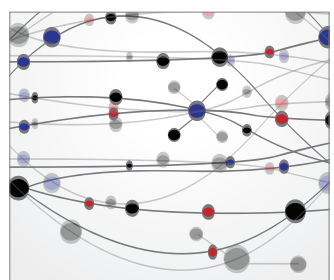

\section{The Scientific} World Journal
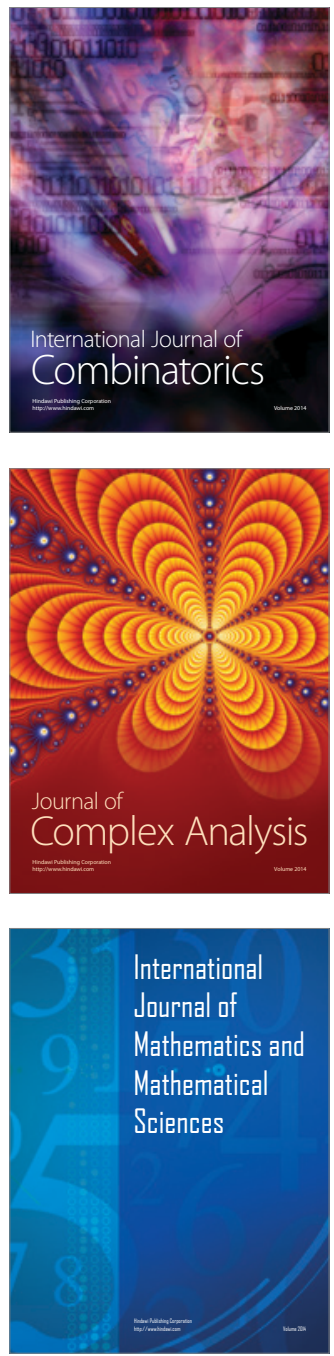
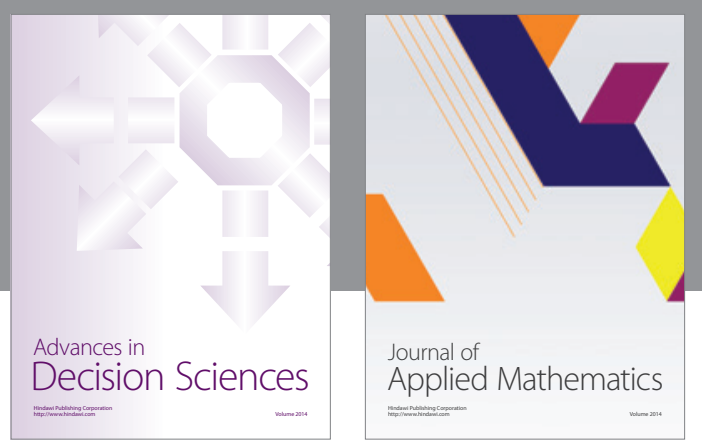

Algebra

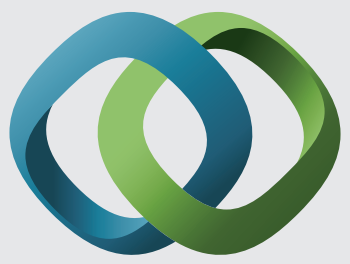

\section{Hindawi}

Submit your manuscripts at

https://www.hindawi.com
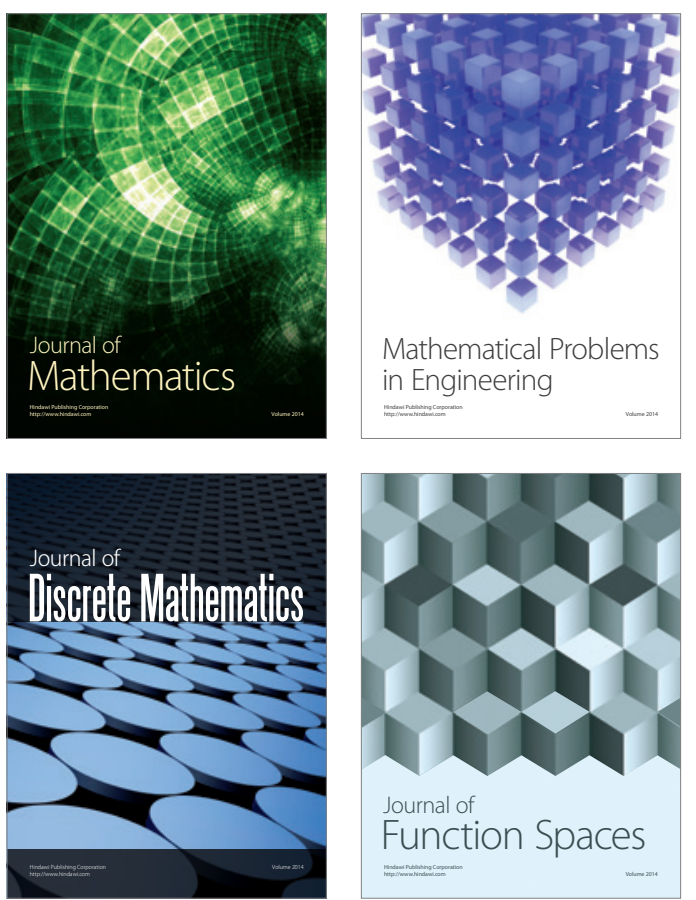

Mathematical Problems in Engineering
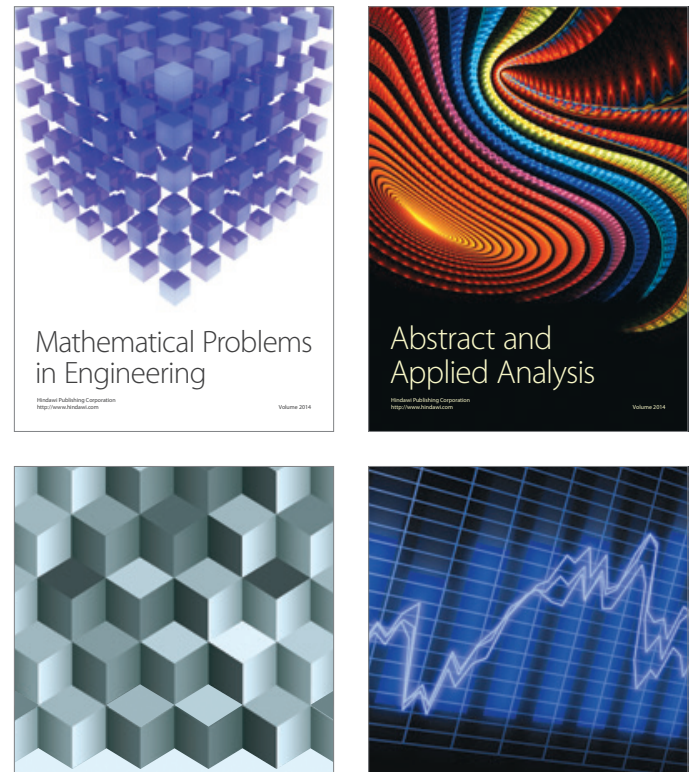

Journal of

Function Spaces

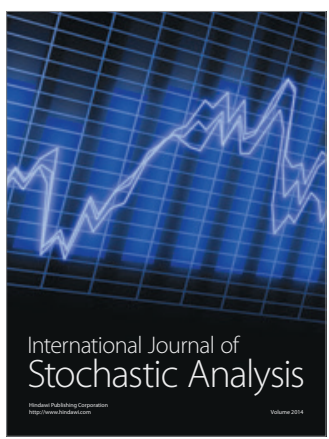

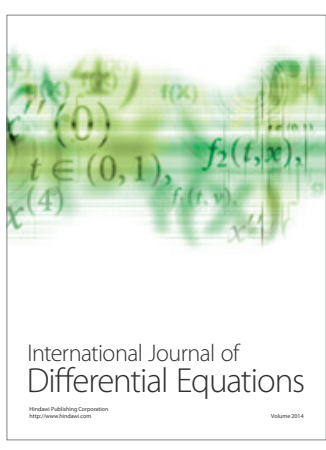
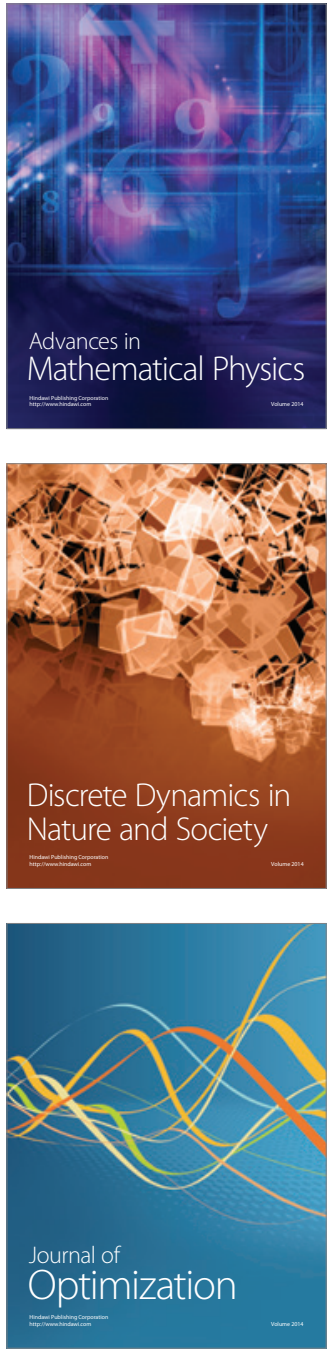